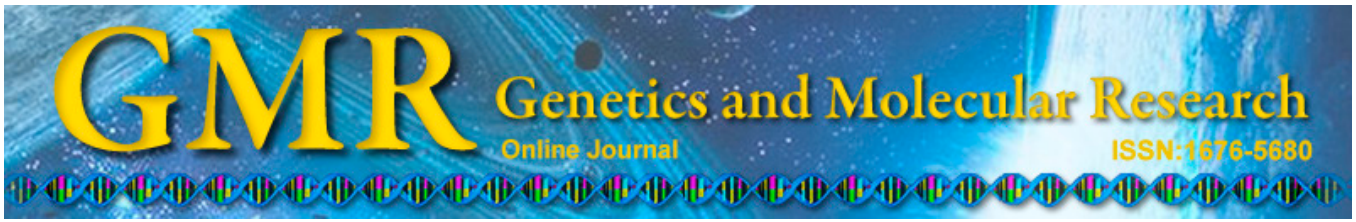

\title{
CYP4F2 gene single nucleotide polymorphism is associated with ischemic stroke
}

\author{
H.-Q. Yan ${ }^{1}$, Y. Yuan ${ }^{1}$, P. Zhang ${ }^{1}$, Z. Huang ${ }^{1}$, L. Chang ${ }^{1}$ and Y.-K. Gui ${ }^{1}$ \\ Department of Neurology, First Affiliated Hospital of Xinxiang Medical College, \\ Xinxiang, China \\ Corresponding author: H.-Q. Yan \\ E-mail: yanhai_qing@yeah.net
}

Genet. Mol. Res. 14 (1): 659-664 (2015)

Received June 24, 2014

Accepted November 18, 2014

Published January 30, 2015

DOI http://dx.doi.org/10.4238/2015.January.30.8

\begin{abstract}
The objective of this study was to explore the relationship between CYP4F2 gene polymorphism and ischemic stroke (IS) in the Han Chinese population. We performed a case-control study to genotype four single nucleotide polymorphisms (SNPs) (rs2108622, rs3093100, rs3093105, rs3093135) in the CYF4F2 gene. The genotype and haplotype distributions were compared between the case and control groups. We found that the GG genotype of rs2108622 in the CYP4F2 gene was associated with risk of IS $(\mathrm{P}=0.023)$. Haplotype analysis indicated that the GGGT haplotype comprising rs2108622rs3093100-rs3093105-rs3093135 was associated with IS, which suggests that the GGGT haplotype may be a risk factor for IS ( $\mathrm{P}=$ 0.012). CYP4F2 gene polymorphism might increase the risk of IS in the Chinese population.
\end{abstract}

Key words: Chinese population; $C Y P 4 F 2$ gene; Haplotype; Ischemic stroke; Single nucleotide polymorphism 


\section{INTRODUCTION}

Ischemic stroke (IS) is one of the most common diseases to affect human health and its morbidity and mortality rates are very high (Cha et al., 2014). Previous studies have indicated that IS is a complex polygenic disease resulting from the interaction between genetic variation and environmental factors (Criado-García et al., 2011). Although the exact genetic mechanism is unclear, some new IS susceptibility genes have recently been reported, such as the apolipoprotein (Apo) E gene (Gu et al., 2013), the phosphodiesterase (PDE) 4D gene (Heyer et al., 2013), the ALOX5AP gene (Ye et al., 2014), and the interleukin (IL) 1 gene (Luo et al., 2013). However, these genes have not been reported in all ethnicities. CYP4F2 is expressed in the liver, heart, lung, kidney, and white blood cells, and is involved in 20-hydroxy eicosane arachidonic acid (20-HETE) metabolism (Fava et al., 2012). 20-HETE in vivo has significant physiological effects and achieves vascular smooth muscle depolarization through blocking $\mathrm{Ca}^{2+} \mathrm{ac}^{-}$ tivation and $\mathrm{K}^{+}$channels, resulting in strong vasoconstriction. 20-HETE plays an important role in the regulation of renal, cerebral, coronary vasoconstriction, and pulmonary circulation $(\mathrm{Wu}$ et al., 2014). Animal experiments have shown that 20-HETE can cause vasospasm after hemorrhagic stroke, and it participates in the incidence and development of ischemic stroke (Kehl et al., 2002). When 20-HETE synthesis is suppressed in a cerebral infarction rat model, infarct size can be reduced (Imaoka et al., 2005). In a hypertensive rat model, the increased 20-HETE production can lead to oxidative stress and endothelial cell damage, resulting in increased incidence of IS (Williams et al., 2012). CYP4F2 is the major synthase that catalyzes arachidonic acid to generate 20-HETE. Recently, Stec et al. (2007) demonstrated that a single nucleotide polymorphism (SNP) (rs2108622, V433M) in the CYP4F2 gene can cause 20-HETE reduction from arachidonic acid. Fava et al. (2008) studied elderly patients with cerebral infarction in Sweden and found that the V433M mutation in the CYP4F2 gene is associated with cerebral infarction in male patients. A study based on the Japanese population (Nakamura et al., 2012) found that $\mathrm{G}$ allele frequency of rs 2108622 in male patients was higher than that in the controls. The TCG haplotype comprising rs3093135-rs1558139-rs2108622 is a risk factor for IS in men (Fu et al., 2009). However, the relationship between CYP4F2 genetic polymorphisms and IS in the Chinese population remains unclear. In the present study, we utilized a case-control study to reveal the relationship between $C Y P 4 F 2$ gene polymorphism and IS.

\section{MATERIAL AND METHODS}

The present study was approved by the Ethics Committee of the First Affiliated Hospital of Xinxiang Medical College and was conducted according to the standards of the Declaration of Helsinki. Written informed consent was obtained from the participants.

\section{Subjects}

A total of 310 hospitalized IS patients (190 male and 120 female) in the Department of Neurology, First Affiliated Hospital of Xinxiang Medical College were enrolled from September 2010 to April 2012. The average age was $57.8 \pm 13.3$ years. All the patients were unrelated Han Chinese people. The patients were diagnosed with IS according to the criteria of the 9th Edition of International Classification of Diseases (ICD9), and all patients underwent magnetic resonance imaging (MRI) or computerized tomography (CT) of the head to confirm 
the diagnosis. Patients with cerebral hemorrhage, subarachnoid hemorrhage, collagen disease, cerebral infarction caused by vascular inflammation, amyloidosis, arteriovenous malformations, and other diseases were excluded from this study.

During the same period, 330 healthy people in the medical center of the same hospital were selected as the control group. All the control subjects were unrelated Han people whose ages and genders were matched with the patient group. There were 211 males and 119 females whose ages ranged from 35 to 87 (58.5 \pm 11.1$)$ years. Subjects with cerebrovascular diseases, neurological diseases, kidney disease, blood disorders, cancer, peripheral vascular diseases, and autoimmune diseases were excluded from the control group. The control subjects had no history of cerebrovascular disease and no sign of cerebrovascular disease was revealed by CT or MRI scanning. Clinical characteristics, including age, gender, height, weight, blood pressure, lipids profiles, fasting glucose, past medical history, drug use history, smoking history, and alcohol consumption history were collected.

\section{DNA extraction}

Fasting venous blood $(2 \mathrm{~mL})$ was taken from the antecubital vein and placed in ethylenediaminetetraacetic acid (EDTA)-containing tubes. A genomic DNA extraction kit (Promega Corporation, United States) was used for DNA extraction from blood samples of the subjects according to the kit protocol.

\section{SNPs selection}

We searched tag SNP in the human HapMap Project database with the following criteria: $\mathrm{r}^{2} \geq 0.5$ and minimum allele frequency $\geq 0.1$, and we found four tag SNPs (rs3093135, rs2108622, rs3093100, and rs1558139) in the CYP4F2 gene.

\section{Genotyping}

We utilized polymerase chain reaction-restriction fragment length polymorphism to genotype the four SNPs. The primers were designed using the design software primer 5.0. The primers are shown in Table 1.

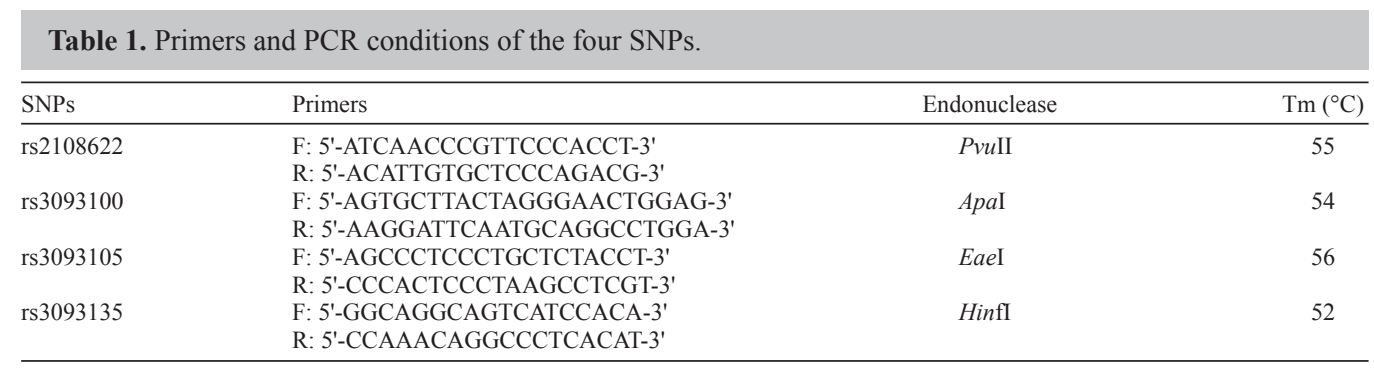

\section{Statistical analysis}

We utilized the SPSS 13.0 statistical software (Chicago, IL, USA) to perform the 
data analyses. The Hardy-Weinberg (H-W) equilibrium test was carried out by the chi-square test. The continuous data were compared using the $t$-test, and categorical data were compared using the chi-square test. Genotype and allele frequencies were compared using the chisquare test. Non-conditional logistic regression was used to adjust the traditional risk factors for stroke such as gender, age, body mass index, blood pressure, blood lipids, blood glucose, smoking history, history of alcohol consumption, and other confounding factors. The odds ratios (ORs) and 95\% confidence intervals (CIs) were calculated before and after adjustment. We utilized the SHEsis software to perform the haplotype analyses. $\mathrm{P}<0.05$ was considered to be significant.

\section{RESULTS}

\section{Characteristics of participants}

The distribution of age, body mass index, high-density lipoprotein cholesterol, and low-density lipoprotein cholesterol showed no significant differences between the IS group and the control group. There were significant differences between the IS group and the control in hypertension, diabetes, smoking history, diastolic blood pressure, systolic blood pressure, and glucose, triglycerides, and total cholesterol concentrations (Table 2).

\begin{tabular}{|c|c|c|c|c|c|c|c|c|c|c|c|c|c|}
\hline Groups & $\mathrm{N}$ & $\begin{array}{c}\text { Age } \\
\text { (years) }\end{array}$ & $\begin{array}{c}\text { BMI } \\
\left(\mathrm{kg} / \mathrm{m}^{2}\right)\end{array}$ & $\begin{array}{c}\mathrm{SBP} \\
(\mathrm{mmHg})\end{array}$ & $\begin{array}{c}\text { DBP } \\
(\mathrm{mmHg})\end{array}$ & $\begin{array}{c}\text { Hypertension } \\
{[\mathrm{N}(\%)]}\end{array}$ & $\begin{array}{l}\text { Diabetes } \\
{[\mathrm{N}(\%)]}\end{array}$ & $\begin{array}{l}\text { Smoking } \\
{[N(\%)]}\end{array}$ & $\begin{array}{l}\text { GLU } \\
(\mathrm{mM})\end{array}$ & $\begin{array}{c}\mathrm{TG} \\
(\mathrm{mM})\end{array}$ & $\begin{array}{c}\mathrm{TC} \\
(\mathrm{mM})\end{array}$ & $\begin{array}{c}\text { HDL-C } \\
(\mathrm{mM})\end{array}$ & $\begin{array}{c}\text { LDL-C } \\
(\mathrm{mM})\end{array}$ \\
\hline \multicolumn{14}{|l|}{ Total } \\
\hline IS & 310 & $57.8 \pm 13.3$ & $25.4 \pm 3.4$ & $142.1 \pm 25.1$ & $88.4 \pm 16.1$ & $112(36.1)$ & $104(33.5)$ & $87(28.1)$ & $7.7 \pm 3.9$ & $3.3 \pm 2.8$ & $5.3 \pm 2.4$ & $1.2 \pm 0.8$ & $2.9 \pm 1.3$ \\
\hline Control & 330 & $58.5 \pm 11.1$ & $24.8 \pm 3.5$ & $124.4 \pm 14.5$ & $78.4 \pm 14.3$ & $44(13.3)$ & $23(7.0)$ & $49(14.9)$ & $4.8 \pm 1.3$ & $1.2 \pm 1.1$ & $4.1 \pm 1.1$ & $1.4 \pm 0.5$ & $2.1 \pm 0.9$ \\
\hline$P$ & & 0.322 & 0.053 & $<0.001$ & $<0.001$ & $<0.001$ & 0.001 & $<0.001$ & $<0.001$ & $<0.001$ & $<0.001$ & 0.114 & 0.123 \\
\hline
\end{tabular}

\section{H-W equilibrium}

The genotype distribution in all SNPs were in line with the H-W genetic equilibrium in both the IS and the control groups (all $\mathrm{P}>0.05$, data not shown).

\section{Genotype and allele frequencies}

We found the GG genotype frequency of rs 2108622 was significantly higher in the IS patients than in the control group $(\mathrm{P}=0.023)$, and the $\mathrm{G}$ allele frequency was also significantly higher than that in control group $(\mathrm{P}=0.011$, Table 3$)$.

Logistic regression analysis showed that after adjustment of the conventional risk factors such as hypertension, diabetes, hyperlipidemia, and smoking, the difference of GG genotype frequency in male patients and the control group remained statistically significant $(\mathrm{OR}=3.335,95 \% \mathrm{CI}=1.324-5.543, \mathrm{P}=0.008)$. 


\begin{tabular}{|c|c|c|c|c|c|c|c|c|}
\hline \multirow[t]{2}{*}{ SNPs } & \multirow[t]{2}{*}{ Alleles $(1 / 2)$} & \multirow[t]{2}{*}{ Groups } & \multicolumn{3}{|c|}{ Genotypes [N (\%)] } & \multirow[t]{2}{*}{$P$ value } & \multirow[t]{2}{*}{ MAF } & \multirow[t]{2}{*}{$P$ value } \\
\hline & & & $1 / 1$ & $1 / 2$ & $2 / 2$ & & & \\
\hline \multirow{2}{*}{ rs 2108622} & $\mathrm{G} / \mathrm{A}$ & IS & $167(0.54)$ & $118(0.38)$ & $25(0.08)$ & 0.023 & 0.271 & 0.011 \\
\hline & & Control & $152(0.46)$ & $142(0.43)$ & $36(0.11)$ & & 0.324 & \\
\hline \multirow[t]{2}{*}{ rs 3093100} & $\mathrm{G} / \mathrm{C}$ & IS & $239(0.77)$ & $65(0.21)$ & $6(0.02)$ & 0.121 & 0.124 & 0.085 \\
\hline & & Control & $248(0.75)$ & $73(0.22)$ & $10(0.03)$ & & 0.141 & \\
\hline \multirow[t]{2}{*}{ rs3093105 } & $\mathrm{T} / \mathrm{G}$ & IS & $210(0.68)$ & $84(0.27)$ & $16(0.05)$ & 0.323 & 0.187 & 0.076 \\
\hline & & Control & $257(0.78)$ & $66(0.20)$ & $7(0.02)$ & & 0.121 & \\
\hline \multirow[t]{2}{*}{ rs3093135 } & $\mathrm{T} / \mathrm{A}$ & IS & $236(0.76)$ & $65(0.21)$ & $9(0.03)$ & 0.112 & 0.134 & 0.432 \\
\hline & & Control & $244(0.74)$ & $77(0.23)$ & $9(0.03)$ & & 0.144 & \\
\hline
\end{tabular}

SNPs $=$ single nucleotide polymorphisms; IS = ischemic stroke; MAF $=$ minimum allele frequency.

\section{Haplotype analyses}

According to the results of linkage disequilibrium analysis, we chose rs2108622rs3093100-rs3093105-rs3093135 to construct haplotypes using the SHEsis software. The results showed that, in total, GGGT haplotype distribution frequency in the IS group was significantly higher than in the control group. Individuals carrying the GGGT haplotype had 1.559 times increased risk of IS $(\mathrm{OR}=1.559 ; 95 \% \mathrm{CI}=1.022-1.897 ; \mathrm{P}=0.012)$ (Table 4).

Table 4. Haplotype distribution between case and control.
\begin{tabular}{lccccc}
\hline Haplotypes & IS & Control & P & OR & $95 \%$ CI \\
\hline GGGT & 0.554 & 0.484 & 0.012 & 1.559 & $1.022-1.897$ \\
GGGA & 0.044 & 0.062 & 0.098 & 0.753 & $0.322-1.087$ \\
GGTT & 0.075 & 0.081 & 0.212 & 0.865 & $0.654-1.121$ \\
AGGT & 0.155 & 0.177 & 0.443 & 0.768 & $0.353-1.088$ \\
ACTT & 0.041 & 0.034 & 0.383 & 1.432 & $0.988-1.987$ \\
\hline
\end{tabular}

IS = ischemic stroke; $\mathrm{OR}=$ odds ratio; $95 \% \mathrm{CI}=95 \%$ confidence interval.

\section{DISCUSSION}

In the present study, we found that $\mathrm{G}$ allele carriers have a higher risk of IS in the Han Chinese population. The genetic variation is the molecular basis of human genetic diversity. IS is caused by the interaction between environmental and genetic factors. In this study, an important human metabolic enzyme P450 gene family member, the CYP4F2 gene, was selected as the candidate gene to perform the case-control study. We found that the GG genotype of rs2108622 in the CYP4F2 gene was significantly higher in IS patient than in the control group, as was the G allele. We also found that the GGGT haplotype comprising rs2108622rs3093100-rs3093105-rs3093135 was the susceptibility haplotype for IS. Our results are consistent with the results reported by Fu et al. (2009), but inconsistent with results by Ward et al. (2008) and Fava et al. (2008). Ward et al. (2008) found that the A allele was a risk factor for hypertension. The other study by Fava et al. (2012) found that the A allele was a risk factor for IS. This difference may be due to differences in race, differences in methodology, or different patient selection criteria.

Previous studies have shown that androgens can interact with CYPs; Singh and Shwartzman (2008) found that androgen-induced CYP4A8 expression reduced CYP2C23 
expression and caused increased production of 20-HETE, then epoxyeicosatrienoic acid decreased, which affected the contraction of blood vessels. However, the exact mechanism of the susceptibility for IS remains unclear.

\section{CONCLUSION}

This study showed that the GGGT haplotype comprising rs2108622-rs3093100rs3093105-rs3093135 in the CYP4F2 gene is associated with IS in Han Chinese men.

\section{Conflicts of interest}

The authors declare no conflit of interest.

\section{REFERENCES}

Cha MJ, Park H, Lee MH, Cho Y, et al. (2014). Prevalence of and risk factors for silent ischemic stroke in patients with atrial fibrillation as determined by brain magnetic resonance imaging. Am. J. Cardiol. 113: 655-661.

Criado-García J, Fuentes F, Cruz-Teno C, García-Rios A, et al. (2011). R353Q polymorphism in the factor vii gene and cardiovascular risk in heterozygous familial hypercholesterolemia: a case-control study. Lipids Health Dis. 10: 50.

Fava C, Montagnana M, Almgren P, Rosberg L, et al. (2008). The V433M variant of the CYP4F2 is associated with ischemic stroke in male Swedes beyond its effect on blood pressure. Hypertension 52: 373-380.

Fava C, Ricci M, Melander O and Minuz P (2012). Hypertension, cardiovascular risk and polymorphisms in genes controlling the cytochrome $\mathrm{P} 450$ pathway of arachidonic acid: a sex-specific relation? Prostaglandins Other Lipid Mediat. 98: 75-85.

Fu Z, Nakayama T, Sato N, Izumi Y, et al. (2009). A haplotype of the CYP4F2 gene associated with myocardial infarction in Japanese men. Mol. Genet. Metab. 96: 145-147.

$\mathrm{Gu}$ L, Su L, Chen Q, Liang B, et al. (2013). Association between the apolipoprotein E gene polymorphism and ischemic stroke in Chinese populations: new data and meta-analysis. Exp. Ther. Med. 5: 853-859.

Heyer EJ, Mergeche JL, Ward JT, Malone HR, et al. (2013). Phosphodiesterase 4D single-nucleotide polymorphism 83 and cognitive dysfunction in carotid endarterectomy patients. Neurosurgery 73: 791-796.

Imaoka S, Hashizume T and Funae Y (2005). Localization of rat cytochrome P450 in various tissues and comparison of arachidonic acid metabolism by rat P450 with that by human P450 orthologs. Drug Metab. Pharmacokinet. 20: 478-484.

Kehl F, Cambj-Sapunar L, Maier KG, Miyata N, et al. (2002). 20-HETE contributes to the acute fall in cerebral blood flow after subarachnoid hemorrhage in the rat. Am. J. Physiol. Heart Circ. Physiol. 282: H1556-1565.

Luo S, Wang F, Li Z and Deng J (2013). Effect of the $+781 \mathrm{C} / \mathrm{T}$ polymorphism in the interleukin- 8 gene on atherosclerotic cerebral infarction, and its interaction with smoking and drinking. PLoS One 8: e80246.

Nakamura K, Obayashi K, Araki T, Aomori T, et al. (2012). CYP4F2 gene polymorphism as a contributor to warfarin maintenance dose in Japanese subjects. J. Clin. Pharm. Ther. 37: 481-485.

Singh H and Schwartzman ML (2008). Renal vascular cytochrome P450-derived eicosanoids in androgen-induced hypertension. Pharmacol. Rep. 60: 29-37.

Stec DE, Roman RJ, Flasch A and Rieder MJ (2007). Functional polymorphism in human CYP4F2 decreases 20-HETE production. Physiol. Genomics 30: 74-81.

Ward NC, Tsai IJ, Barden A, van Bockxmeer FM, et al. (2008). A single nucleotide polymorphism in the CYP4F2 but not CYP4A11 gene is associated with increased 20-HETE excretion and blood pressure. Hypertension 51: 1393-1398.

Williams JM, Fan F, Murphy S, Schreck C, et al. (2012). Role of 20-HETE in the antihypertensive effect of transfer of chromosome 5 from Brown Norway to Dahl salt-sensitive rats. Am. J. Physiol. Regul. Integr. Comp. Physiol. 302: R1209-18.

Wu CC, Gupta T, Garcia V, Ding Y, et al. (2014). 20-HETE and blood pressure regulation: clinical implications. Cardiol. Rev. 22: 1-12.

Ye F, Liu NN, Zheng YQ, Zhang WJ, et al. (2014). Three polymorphisms of ALOX5AP and risk of ischemic stroke in Chinese: evidence from a meta-analysis. J. Neurol. Sci. 336: 93-98. 